\title{
Long spin diffusion lengths in doped conjugated polymers due to enhanced exchange coupling
}

Shu-Jen Wang $^{1+}$, Deepak Venkateshvaran ${ }^{1{ }^{+*}}$, M. R. Mahani ${ }^{2}$, Uday Chopra ${ }^{2,3}$, Erik R. McNellis ${ }^{2}$, Riccardo Di Pietro ${ }^{4}$, Sam Schott ${ }^{1}$, Angela Wittmann ${ }^{1}$, Guillaume Schweicher ${ }^{1}$, Murat Cubukcu ${ }^{1}$, Keehoon Kang ${ }^{1}$, Remington Carey ${ }^{1}$, Thomas J. Wagner ${ }^{4}$, Janis N. M. Siebrecht ${ }^{1}$, Daniel P. G. H. Wong $^{1}$, Ian E. Jacobs ${ }^{1}$, Razan O. Aboljadayel ${ }^{5}$, Adrian lonescu ${ }^{5}$, Sergei A. Egorov ${ }^{2,6,7}$, Sebastian Mueller ${ }^{2}$, Olga Zadvorna ${ }^{1}$, Piotr Skalski ${ }^{1}$, Cameron Jellett ${ }^{8}$, Mark Little $^{8}$, Adam Marks ${ }^{8}$, lain McCulloch $^{8}$, Joerg Wunderlich ${ }^{4,9}$, Jairo Sinova ${ }^{2}$, and Henning Sirringhaus ${ }^{1 *}$

${ }^{1}$ Optoelectronics Group, Cavendish Laboratory, JJ Thomson Avenue, University of Cambridge, CB3 OHE, Cambridge, United Kingdom

${ }^{2}$ Institute of Physics, INSPIRE Group, Johannes Gutenberg Universität, Staudingerweg 7, Mainz, 55128 Germany

${ }^{3}$ Graduate School Material Science in Mainz, Staudingerweg 9, 55128 Mainz, Germany ${ }^{4}$ Hitachi Cambridge Laboratory, JJ Thomson Avenue, CB3 OHE, Cambridge, United Kingdom ${ }^{5}$ Thin Film Magnetism Group, Cavendish Laboratory, JJ Thomson Avenue, University of Cambridge, $\mathrm{CB} 3 \mathrm{OHE}$, Cambridge, United Kingdom

${ }^{6}$ University of Virginia, Chemistry Department, McCormick Rd, Charlottesville, VA 22901 USA

${ }^{7}$ Leibniz Institute for Polymer Research, Dresden, Hohe Strasse 6, D-01069 Dresden, Germany ${ }^{8}$ Department of Chemistry, Imperial College, SW7 2AZ, London, United Kingdom ${ }^{9}$ Institute of Physics, Academy of Sciences of the Czech Republic, Praha 6, Czech Republic

these authors contributed equally

*correspondence: $\underline{\text { dv246@cam.ac.uk, hs220@cam.ac.uk }}$ 
Carbon-based semiconductors such as conjugated organic polymers are of potential use in the development of spintronic devices and spin-based information processing. In particular, these materials offer a low spin-orbit coupling strength due to their relatively light constituent chemical elements, which should, in principle, favour long spin diffusion lengths. However, organic polymers are relatively disordered materials and typically have a carrier mobility that is orders of magnitude lower than crystalline inorganic materials. As a result, small spin diffusion lengths of around $50 \mathrm{~nm}$ have typically been measured using vertical organic spin valves. Here we report measuring spin diffusion lengths in doped conjugated polymers using a lateral spin transport device architecture, which is based on spin pumping injection and inverse spin Hall detection. The approach suggests that long spin diffusion lengths of more than $1 \mu \mathrm{m}$ and fast spin transit times of around $10 \mathrm{~ns}$ are possible in conjugated polymer systems when they have a sufficiently high spin density (around $10^{20} \mathrm{~cm}^{-3}$ ). We explain these results in terms of an exchange-based spin diffusion regime in which the exchange interactions decouple spin and charge transport.

Organic semiconductors have traditionally been used in applications such as energy efficient organic light emitting diode (OLED) displays and printed flexible circuits. However, they are now also increasingly of interest in spintronics ${ }^{1,2}$. This is due, in part, to their low spin orbit coupling (SOC) strength, which is the result of their composition from lighter chemical elements such as carbon ${ }^{3,4}$. In contrast, inorganic semiconductors, such as silicon or GaAs, consist of elements with larger atomic numbers and give rise to stronger spin-orbit coupling; spin-flip scattering induced by strong spin-orbit coupling is known to limit the distance over which spin information and spin currents can be propagated ${ }^{5}$. The underlying mechanism for the propagation of spin currents in organic semiconductors is also expected to be distinct to conventional crystalline inorganic semiconductors ${ }^{6,7,8}$. With inorganic semiconductors, spin transport accompanies charge carriers as they are transported as delocalised Bloch electrons, maintaining coherence during both charge and spin transport ${ }^{9}$. Organic polymers on the other hand are relatively disordered materials where carrier transport takes place via intermolecular hopping between localised states and carrier mobilities and diffusion coefficients are orders of magnitude lower than in crystalline, inorganic materials ${ }^{10}$. 
Spin transport in organic semiconductors has traditionally been studied by measuring the magnetoresistance in trilayer spin valves, where the organic semiconductor is sandwiched between two ferromagnetic layers $11,12,13,14,15,16$. The length scale over which spin information is transported - the spin diffusion length - can be extracted by varying the thickness of the organic spacer layer and mapping the decay of the corresponding magnetoresistance signal. With this approach, typical spin diffusion lengths of around $50 \mathrm{~nm}$ have been extracted for many of these undoped organic semiconductors. These values are disappointingly small compared to inorganic semiconductors such as GaAs, Ge and Si in which values exceeding $1 \mu \mathrm{m}$ have been achieved ${ }^{9}, 17,18$. Even larger spin diffusion lengths of between $30 \mu \mathrm{m}$ and $130 \mu \mathrm{m}$ have also been reported in Graphene ${ }^{19,20}$.

In principle, the low SOC strength in organic materials favours long spin diffusion lengths. However, the relatively low carrier mobility of most organic semiconductors is expected to strongly limit the achievable spin diffusion lengths ${ }^{21}$. The dominant cause of spin relaxation in low charge carrier mobility polymers is attributed to hyperfine interactions due to hydrogen atoms within the molecule and can be suppressed with the use of deuteration ${ }^{12}$. Furthermore, it has been postulated theoretically that in organic systems where the carrier (or spin) concentration is sufficiently high, spin diffusion could potentially be possible over long distances despite low carrier mobilities ${ }^{8}$. However, no direct experimental evidence supporting such a regime has been demonstrated so far. In such a regime with highly mobile spins it is also expected that the spin-orbit coupling will be more dominant than hyperfine interactions.

Measurements of spin diffusion lengths in organic spin valves are now relatively routine. However, the majority of reported devices use Lanthanum Strontium Manganite (LSMO) as one of the ferromagnets in the trilayer stacks. LSMO is known to have a strong bi-axial anisotropy and it is now clear that some observations of a magnetoresistance signal in organic spin valves may have arisen from the phenomenon of Tunnelling Anisotropic Magnetoresistance (TAMR) within the LSMO contact ${ }^{22}$. It was also recently demonstrated that a reduction in the junction area of an organic spin valve causes the measured magnetoresistance to vanish, revealing the role pinholes play in large area organic spin valves 23; the presence of pinholes within the organic spacer makes it difficult to distinguish between diffusive and ballistic spin transport in organic devices. Such ambiguities call into question the 
accuracy of many reported trilayer spin valve results and highlight the need for alternative artefact-free approaches to probing spin transport in organic semiconductors.

A solid-state approach for measuring spin diffusion in semiconductors that is generally considered unambiguous is to use nonlocal lateral device architectures - a method that allows spin diffusion to be decoupled from spin drift within the same device $9,24,25$. In this setup, pure diffusive spin transport is probed by measuring the spin accumulation above or below a ferromagnetic detector electrode in comparison to an Ohmic contact far away from the detector where the spin accumulation has completely relaxed. Such nonlocal spin valve (NLSV) measurements have successfully quantified spin diffusion lengths in Graphene and in inorganic materials such as Ge, GaAs and in various metals $26,27,9,28,24,29,30,31$. Unfortunately, in conjugated organic semiconductors, a nonlocal spin valve signal has never been successfully measured despite multiple diligent attempts. We have unsuccessfully attempted to perform all-electrical spin injection and spin detection measurements on conjugated polymers using various nonlocal architectures, in which spin detection is achieved through either a spin accumulation or a spin current measurement. The failure of these techniques is, we believe, due to a combination of conductance mismatch at the organic semiconductor / ferromagnetic metal interface ${ }^{32}$ and large voltage noise fluctuations on the measured nonlocal voltage. The latter arise due to the fundamental nature of stochastic hopping transport [1/f noise] in these disordered materials as charge current spreads within the active layer from the injector, rendering nonlocal measurements extremely challenging if not impossible $33,34,35$. A detailed discussion of our attempts to perform all-electrical spin injection and detection experiments in nonlocal geometries is provided in Supplementary Information (SI) Sections 1-3.

In this Article, in order to circumvent the roadblocks associated with electrical spin injection into organic semiconductors using trilayer and nonlocal architectures, we examine spin injection into doped conjugated polymers using an experimental technique based on spin pumping. This technique involves the dissipation of angular momentum at a ferromagnetic injector and into a non-magnetic metal or semiconductor layer and achieves spin injection without charge injection across an interface $36,37,38,39,40,41$. Using this approach, we obtain reliable observations of very long spin diffusion lengths of more than $1 \mu \mathrm{m}$ in conjugated polymer systems in which spin diffusion is enhanced by exchange effects. 


\section{Pumping, transport and detection of spins in doped organic polymers}

Figure 1(a) shows a schematic of our lateral spin pumping architecture and measurement configuration. Such a lateral spin pumping architecture was never previously demonstrated in organic semiconductors, but was successfully shown in $\mathrm{p}-\mathrm{Si}^{42}, \mathrm{n}-\mathrm{GaAs}{ }^{43}$, Graphene ${ }^{44}, \mathrm{n}-\mathrm{Ge}$ ${ }^{45}, \mathrm{Cu}^{46}$, and a $2 \mathrm{DEG}{ }^{47}$. The device consists of an island of a ferromagnetic permalloy (Py) layer deposited next to a narrow, $1 \mu \mathrm{m}$ wide platinum (Pt) wire running parallel to an edge of the Py island, both of which are in contact with an organic semiconductor. The Py layer is driven into ferromagnetic resonance (FMR) by an applied microwave field in the presence of an external magnetic field and dissipates spin angular momentum into the adjacent organic semiconductor in the form of a spin current $5,41,37,38,48,49$. The injected pure spin current propagates through the organic semiconductor and is absorbed in the Pt wire situated within a few hundred nanometres of the Py island. The absorbed spin current in the Pt detector is finally converted into an electromotive force via the inverse spin Hall effect (ISHE) in Pt and is measured as a voltage $V_{\text {ISHE }}$ across the platinum detector ${ }^{5,50,51}$.

The conjugated polymer in our experiments is poly(2,5-bis(3-alkylthiophen-2-yl)thieno[3,2b]thiophene) (PBTTT) doped with 2,3,5,6-tetrafluoro-7,7,8,8-tetracyanoquinodimethane (F4TCNQ), a widely investigated model system. PBTTT is a semicrystalline conjugated polymer with a highly ordered lamellar microstructure, in which the polymer chains and the direction of $\pi-\pi$ stacking are oriented in the plane of the film and the aliphatic side chains approximately normal to the film plane giving rise to a relatively high, in-plane charge carrier mobility ${ }^{52,53}$. The p-type molecular dopant, F4TCNQ, can be incorporated by a solid-state diffusion process or a sequential doping method that preserves the lamellar ordering and allows reproducible control of the carrier and spin concentration ${ }^{54}$. The dimensions of the device are illustrated in the optical image [Figure 1(b)] with the well resolved sub-micron gap (300 nm in this case) between Py and Pt shown in the SEM image [Figure 1(c)]. Initial measurements were performed inside an ESR cavity, where it was possible to measure the microwave absorption in Py and the ISHE voltage signal in Pt simultaneously. Figure 1(d) shows the microwave absorption signal of the $400 \times 600 \mu \mathrm{m}^{2} \mathrm{Py}$ island on a complete device measured at $9.38 \mathrm{GHz}$ inside the electron spin resonance (ESR) cavity. Fig $1(\mathrm{e})$ is the 
corresponding voltage response measured across the Pt stripe when the Py island is brought into FMR within the ESR cavity. A clean ISHE signal is observed that peaks at the FMR resonance.

a
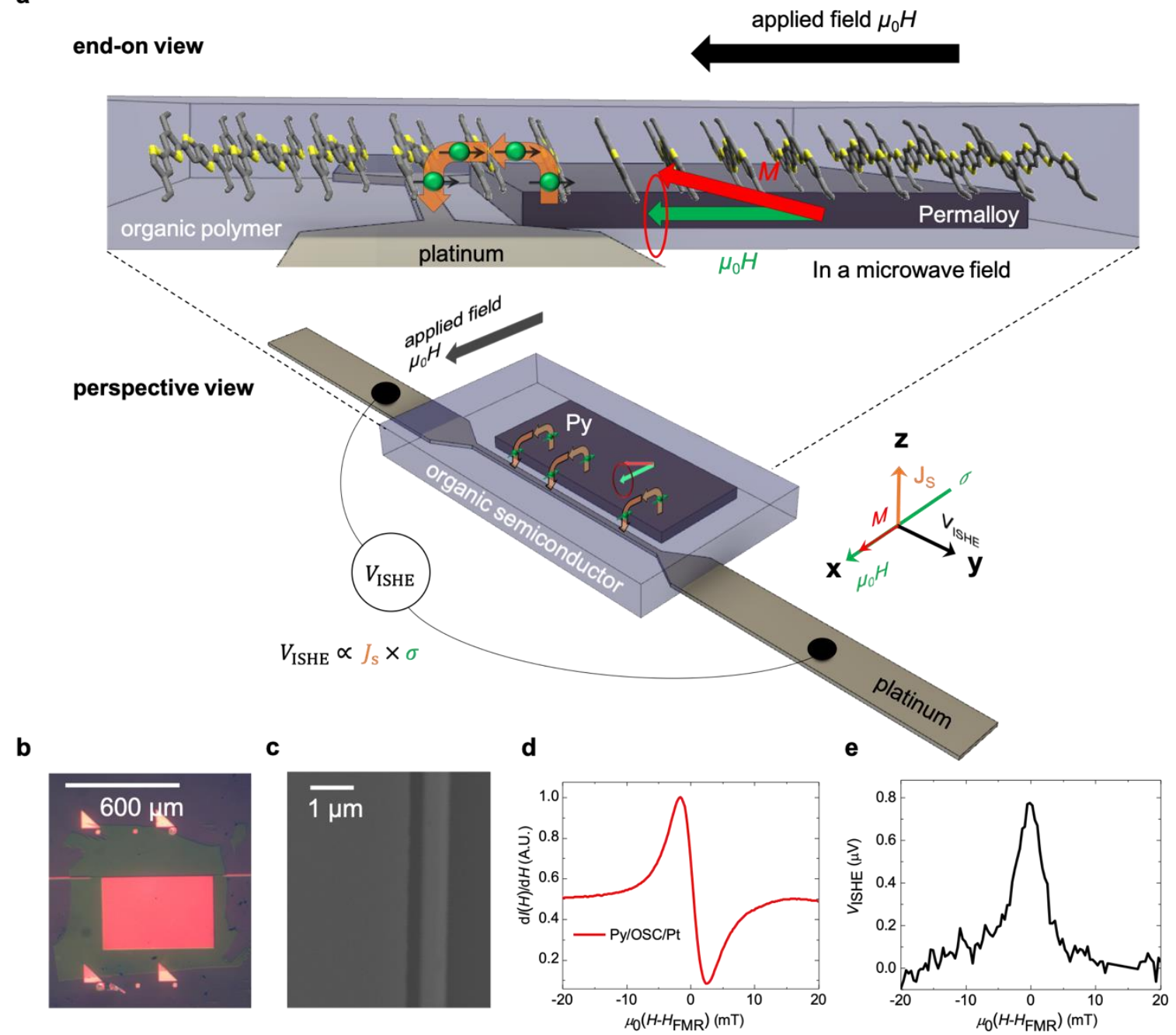

Figure 1 | Lateral spin pumping device architecture and measurement scheme. (a) Schematic illustration of lateral spin pumping and ISHE detection within the nanofabricated device. $\mu_{0} H, M, J_{s}$ and $V_{\text {ISHE }}$ denote the external magnetic field, dynamic magnetisation, pure spin current and voltage due to ISHE, respectively. $\sigma$ is the direction of the spin polarisation and points along $\mu_{0} \mathrm{H}$ as shown on the co-ordinate axis. Enlarged PBTTT molecular faces are shown within the film illustrating spin propagation (orange arrow) down the $\pi$ - $\pi$ stacking direction between molecules (b) Optical image of the lateral spin pumping and ISHE device. (c) SEM image of $300 \mathrm{~nm}$ gap between Py and Pt electrodes. (d) Field $\left(\mu_{0} H\right)$ dependence of FMR signal $d l(H) / d H$ measured for Py/OSC/Pt at $100 \mathrm{~mW}$ microwave excitation and $9.38 \mathrm{GHz}$ microwave frequency inside an ESR cavity. $I$ is the microwave absorption intensity. The external magnetic field was applied in a plane parallel to the film plane, but perpendicular to the long axis of the platinum detector. The FMR field was close to $100 \mathrm{mT}$ under the given excitation conditions. (e) Voltage response picked up by the platinum stripe when the Py island is driven into FMR. 
Figure 2(a) shows the voltage signal detected with an incident microwave power of $1 \mathrm{~W}$ and a frequency of $4 \mathrm{GHz}$ for a device with a gap of $300 \mathrm{~nm}$ between Py and Pt. These measurements, as all subsequent ones, were performed with the sample placed on top of a microwave strip line that provided the FMR excitation. The signal is measured over a long Pt stripe (slightly longer than $600 \mu \mathrm{m}$ ) since the voltage signal scales linearly with length. The measured $V_{\text {ISHE }}$ signal was observed to invert in sign upon reversing the applied in-plane magnetic field direction, consistent with the characteristic symmetry of the inverse spin Hall effect, i.e., $E_{\mathrm{ISHE}} \propto J_{\mathrm{S}} \times \sigma^{51}$. Here, $E_{\mathrm{ISHE}}, J_{\mathrm{s}}$ and $\sigma$ denote the electric field generated by ISHE in the detector, flow of pure spin current, and spin polarisation vector of the spin current, respectively. The latter is aligned with the applied magnetic field. When $\sigma$ was polarised along the direction of the long axis of the Pt detector, $E_{\mathrm{ISHE}}$ was found to vanish (SI Section 4). In a lateral spin pumping device, although there is an electrical contact between the ferromagnetic Py injector and the Pt detector through the doped organic semiconductor, the antisymmetric part of the measured signal due to the anomalous Hall effect (AHE) is reduced in comparison with vertical spin pumping devices on account of the lateral offset between the platinum detector and the permalloy injector.

To fully rule out the AHE contribution from the ferromagnetic layer, as well as potential artefacts due to the anomalous Nernst effect (ANE), and the Nernst effect (NE) of the doped organic semiconductor in the interpretation of the measured signal, a series of careful control experiments were performed. First, the Py island was capped with a $10 \mathrm{~nm} \mathrm{AlO}$ x layer to block spin injection into the organic semiconductor. As shown in the top sub-panel of Figure 2(b), a spin signal at ferromagnetic resonance could not be measured in the presence of the blocking layer. Second, the platinum detector electrode was replaced with metals that have a lower spin-orbit coupling. When the detector electrode was replaced with $\mathrm{Cu}$, no observable signal was measured [Figure 2(b) bottom sub-panel], consistent with the very small spin Hall angle of $\mathrm{Cu}^{5}$. When the detector electrode was replaced with $\mathrm{Au}$, the signal was strongly suppressed [Figure 2(c) top sub-panel] as expected from the spin Hall angle of Au being significantly smaller than that of $\mathrm{Pt}^{5}$. Third, the platinum detector adjacent to the Py island was removed in the region next to the injector as shown in the bottom sub-panel of Figure 2(c). A strong signal could not be observed in this configuration, convincingly ruling out spurious voltage 
contributions due to the ISHE and the Nernst effect of the doped organic semiconductor itself as contributions to our measured signal. These experimental results indicate successful spin transmission in the plane of the F4TCNQ-doped PBTTT film along the polymer backbone and the high mobility $\pi-\pi$ stacking direction. The normalised ISHE induced current, $I_{\text {norm }}=$ $V_{\text {ISHE }} / R$, where $R$ is the measured resistance across the Pt wire, is observed to reduce exponentially with gap spacing between $\mathrm{Py}$ and $\mathrm{Pt}, L_{\mathrm{Py}-\mathrm{Pt}}$, as expected for spin decoherence with distance in the spin transport material [Figure 2(d)]. The spin diffusion length, $\lambda_{s}$, for the sample of F4TCNQ-doped PBTTT is estimated to be $1.2 \pm 0.1 \mu \mathrm{m}$ by fitting $I_{\text {norm }}\left(L_{\mathrm{Py}-\mathrm{Pt}}\right)=$ $I_{0} e^{-L_{\mathrm{Py}-\mathrm{Pt}} / \lambda_{\mathrm{s}}}$ to the experimental data.

a
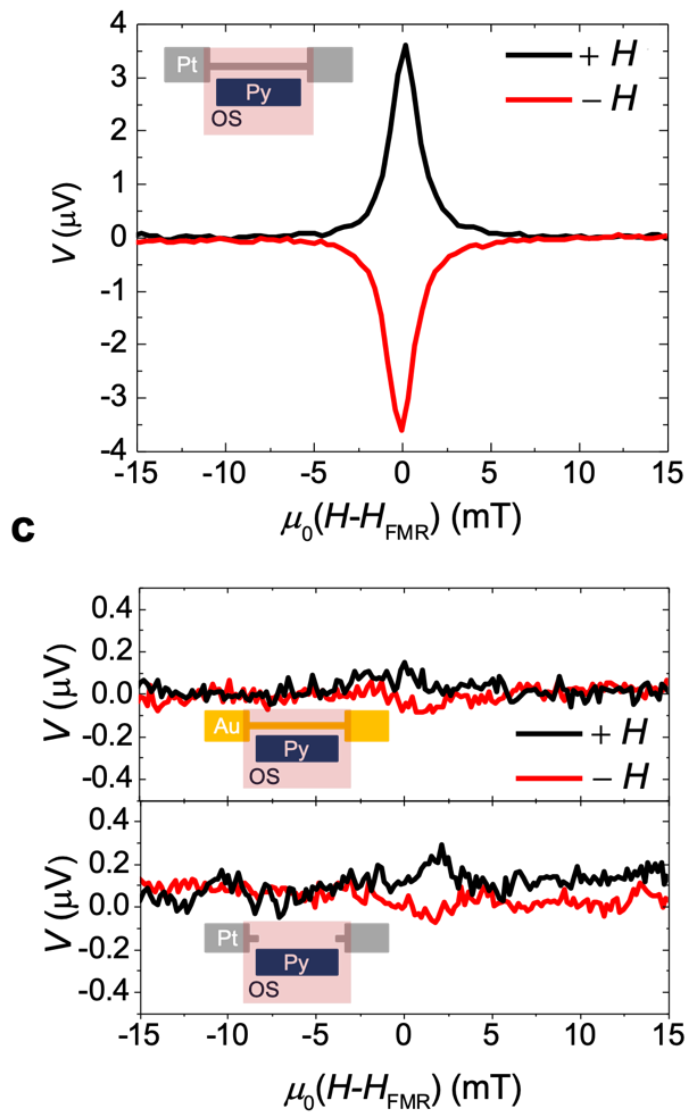

b
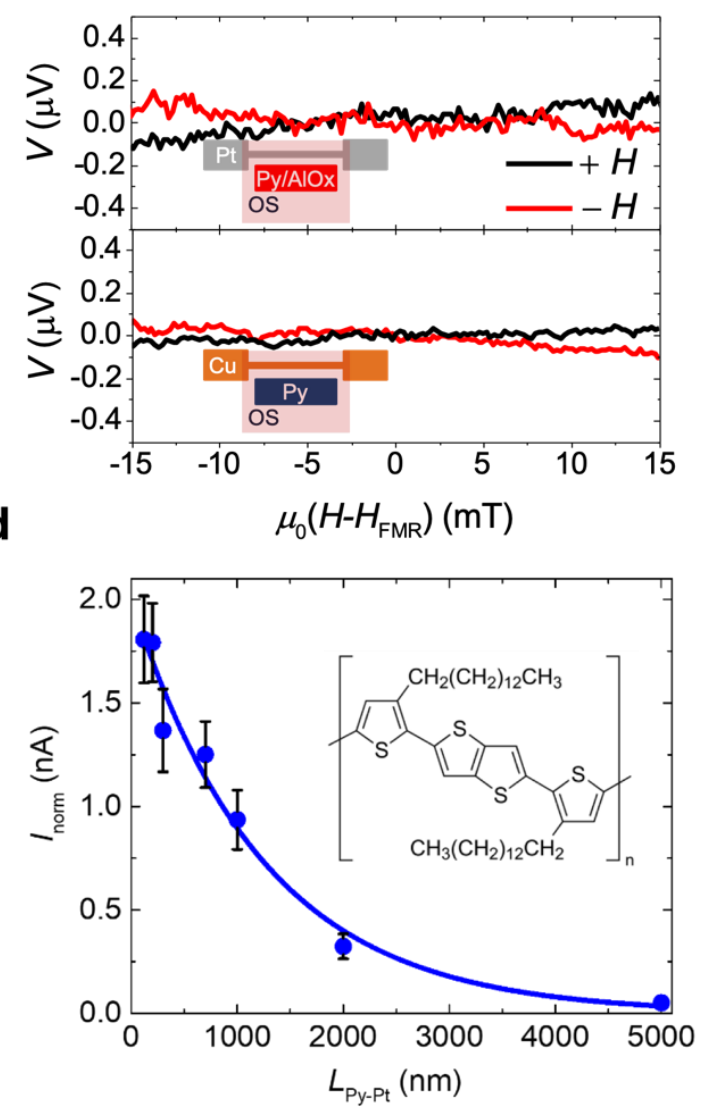

Figure 2 | Observation of long-range spin transport in F4TCNQ-doped PBTTT. Field dependence of the electromotive force $V$ measured across the metal detector for (a) Py $(25 \mathrm{~nm}) / \mathrm{F} 4 T C N Q-P B T T T / P t ~(10 \mathrm{~nm})$, (b) top sub-panel Py $(25 \mathrm{~nm}) / \mathrm{AlO}_{x}(10 \mathrm{~nm}) / \mathrm{F} 4 \mathrm{TCNQ}-\mathrm{PBTTT} / \mathrm{Pt}(10 \mathrm{~nm})$, (b) bottom sub-panel Py $(25 \mathrm{~nm}) / \mathrm{F} 4 \mathrm{TCNQ}$ PBTTT/Cu $(10 \mathrm{~nm})$ (c) top sub-panel Py $(25 \mathrm{~nm}) / \mathrm{F} 4 T C N Q-P B T T T / A u(5 \mathrm{~nm})$, (c) bottom sub-panel Py (25 $\mathrm{nm}$ )/F4TCNQ-PBTTT/nanofabricated broken Pt (10nm). The gap spacing between the Py island and platinum wire was $300 \mathrm{~nm}$ for all devices. The microwave power applied was $1 \mathrm{~W}$ (the microwave power absorbed by the device was around $160 \mu \mathrm{W}$ ) and the microwave frequency was $4 \mathrm{GHz}$. The FMR field ( $H_{\mathrm{FMR}}$ ) was close to $20 \mathrm{mT}$ 
under the given excitation conditions. (d) Gap (between Py and Pt) dependence of ISHE induced current $/$ norm $=$ $V_{\text {ISHE}} / R$. The conductivity of the F4TCNQ-doped PBTTT film was around $100 \mathrm{~S} / \mathrm{cm}$. The blue solid line is an exponential fit.

While performing our control experiments to exclude potential artefacts in the measured spin signal, we also attempted a measurement on a device using $\beta$-Tantalum, a negative spin Hall angle material, as the detector. In using such a negative spin Hall angle detector, an inversion in the polarity of the measured spin signal is expected. Unfortunately, these experiments were unsuccessful and did not produce a measurable signal. We attribute this to the experimental fabrication process in which the organic semiconductor cannot be deposited insitu directly after the deposition of $\beta$-Ta but can only be deposited upon breaking the vacuum and exposing $\beta$-Ta to air, such that due to surface oxidation the purity of $\beta$-Ta at the interface and its negative spin Hall angle property cannot be guaranteed. A detailed account of these experiments is summarised in the supplementary information (SI Section 5).

To better understand the parameters that influence spin transport in F4TCNQ-doped PBTTT, the spin signal measured at the platinum detector was studied as a function of carrier density in the organic semiconductor through its bearing on measured conductivities. Following a procedure to modulate carrier density in doped PBTTT films that our group demonstrated earlier, the carrier density was varied by thermally annealing the fully doped highlyconductive film whereupon the dopant F4TCNQ molecules out-diffuse from PBTTT and the conductivity as well as carrier/spin concentration reduce ${ }^{54}$. As shown in Figure $3(a)$, the measured spin signal /norm was found to decrease very strongly when reducing the carrier concentration (reflected in the conductivities shown). The extracted spin diffusion length within the film at various conductivities is shown in Figure 3(b) and is again clearly seen to decrease with conductivity. Figure 3(c) shows the amplitude of the measured spin signal as a function of temperature in a lateral spin pumping device with a $300 \mathrm{~nm}$ channel and a fully doped $(100 \mathrm{~S} / \mathrm{cm})$ PBTTT film. The spin signal at $50 \mathrm{~K}$ is a factor of three smaller than at 300 K. The conductivity over this temperature range reduces by a factor of four, accompanied by a reduction in both carrier density and mobility by a factor of approximately two each as shown earlier ${ }^{54}$. Compared to the reduction in the spin signal strength due to de-doping (more than a factor of ten upon reduction of the conductivity by a factor of four), the reduction with temperature is weaker. This indicates that the drop in mobility occurring at 
low temperature affects the signal less strongly compared to the reduction in carrier concentration, and highlights the key role that the carrier/spin density plays in mediating spin transport within the polymer.

We have observed similar spin signals in other conjugated polymers. Figure $3(d)$ shows a decaying spin signal with channel length between a Py injector and a Pt detector of a lateral spin pumping device with an active layer of F4TCNQ-doped Poly(3-hexylthiophene-2,5-diyl) (P3HT), which has a similar lamellar, semi-crystalline microstructure as PBTTT. The spin diffusion length extracted for doped P3HT from this data was $565 \pm 60 \mathrm{~nm}$, several hundred $\mathrm{nm}$ shorter than that measured in PBTTT. At its maximum doping concentration, $\mathrm{P} 3 \mathrm{HT}$ has a conductivity of only $3.2 \mathrm{~S} / \mathrm{cm}$ as opposed to $100 \mathrm{~S} / \mathrm{cm}$ in PBTTT. Despite this, P3HT shows a strong spin signal that reduces with conductivity [Figure 3(e)]. It is instructive to note here that the spin density in doped films of PBTTT and P3HT measured using ESR are very similar, with PBTTT having a slightly lower value of $1.6 \times 10^{20} \mathrm{~cm}^{-3}$ in comparison to P3HT having $2.7 \times 10^{20} \mathrm{~cm}^{-3}$. In addition, it is known that on account of a higher crystalline ordering, PBTTT has a higher mobility than P3HT by up to an order of magnitude ${ }^{55}$. Thus, the observation, that both P3HT and PBTTT show a pronounced spin signal despite having a significant difference in their conductivities, points again to the key role that the mobile carrier density and the inter-polaron distance play in spin diffusion. Figure 3(f) shows a plot of the strength of the measured spin signal as a function of measured conductivity for PBTTT, P3HT and another doped conjugated polymer based on cyclopentadithiophenebenzothiadiazole (CDT-BTZ) for a fixed $L_{\mathrm{Py}-\mathrm{Pt}}=300 \mathrm{~nm}$ and provides further confirmation that spin information can be efficiently transported only in polymers with a large carrier density. Similar spin signals could not be measured in devices using undoped polymers. 
a

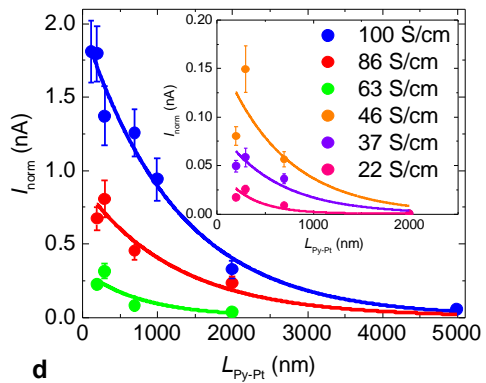

$$
\text { d }
$$

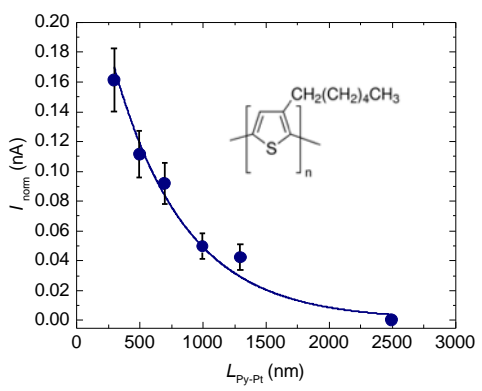

b
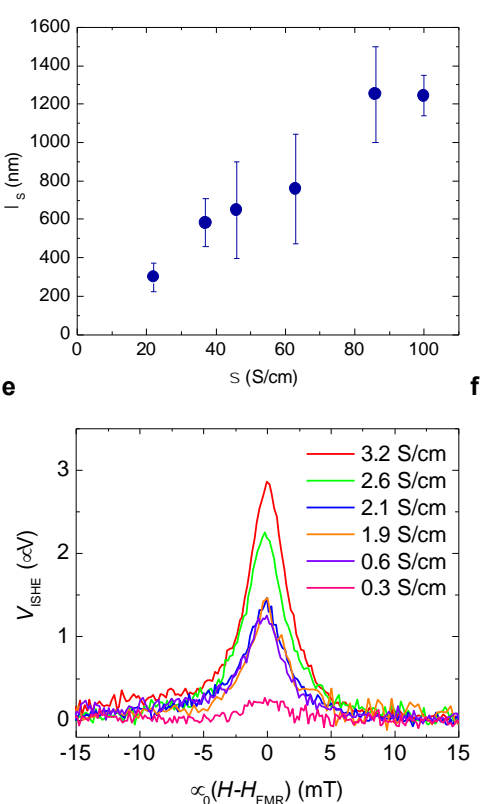

c
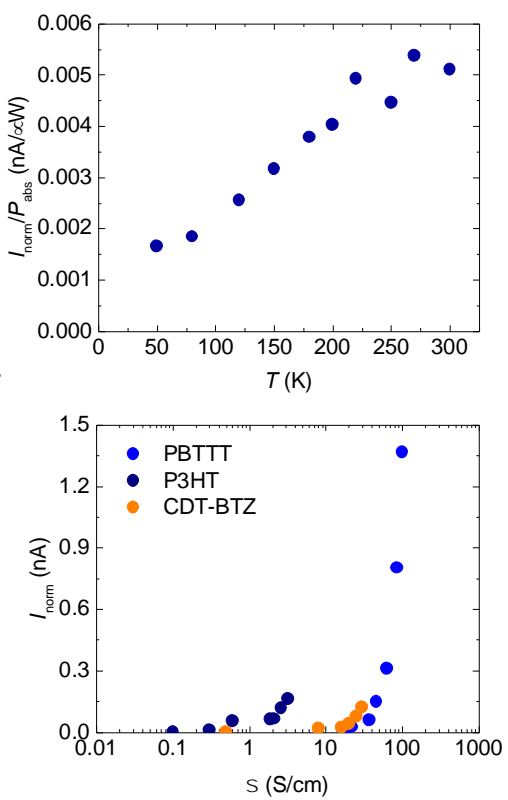

Figure 3 | Carrier density dependence of spin current transport. (a) Gap spacing between Py and Pt dependence of ISHE-induced current with different conductivities of doped F4TCNQ-PBTTT film controlled by thermal annealing. The solid lines are exponential fits to experimental data. (b) Conductivity dependence of spin diffusion length extracted from exponential fits. The error bars indicate the fit uncertainty. (c) Temperature dependence of the spin signal in doped PBTTT. (d) Spin diffusion length extracted from lateral spin pumping in P3HT. (e) Conductivity dependence of the measured spin signal in P3HT. $\mu_{0} H_{\mathrm{FMR}}$ was close to $20 \mathrm{mT}$ (f) Plot of the measured spin signal as a function of conductivity of PBTTT in comparison with P3HT and CDT-BTZ.

We have also investigated the angular dependence of the voltage signal, when tilting the applied magnetic field out-of-plane [Fig. 4(a)]. As the applied magnetic field is rotated out-ofplane by an angle $\theta$, the orientation of magnetisation of the Py island lags by an angle $\theta-\phi$ owing to the shape anisotropy within the film. Using the Landau-Lifshitz-Gilbert (LLG) equation, the magnetisation angle $\phi$ is calculated from the $\theta$ dependence of the resonance field $\left(H_{\mathrm{FMR}}\right)$ shown in Fig. 4(b). The plot of $\phi(\theta)$ in Fig. 4(c) demonstrates how the magnetisation of Py remains relatively in-plane up to $\theta \sim 60^{\circ}$, i.e. magnetisation and field are not collinear, but then reorients quickly as $\theta$ approaches $90^{\circ}$. From this we expect, first of all, the measured ISHE voltage to go through zero and change sign at $\theta=\phi=90^{\circ}$. This is cleanly observed in both PBTTT and P3HT [Fig. 4(d) and 4(e), respectively] and provides further validation of the interpretation of the experiment. The angular dependence also provides interesting information about how the orientation of the injected spin polarisation evolves 
while the spins diffuse through the polymer. Because the FMR-induced polarisation of the spins injected into the organic layer is along the magnetisation direction and the applied field is not collinear with the magnetisation, any reorientation of the spin polarisation due to Hanle-type precession around the applied magnetic field in the polymer, would change the $x$ polarised component of the spin current that reaches the Pt detector [Fig. 1 (a)] and manifest itself in an angular dependence that is significantly more gradual than the simple step-like, $V_{\text {ISHE }} \propto \cos (\phi)$ angular dependence that would be expected if the spin polarisation remained aligned with the magnetisation direction (SI Section 6). In related trilayer spin pumping experiments on undoped PBTTT ${ }^{38}$, silicon ${ }^{50}$, and pentacene ${ }^{56}$, such a gradual angular dependence was claimed to have been observed, but not in $\mathrm{Alq}_{3}{ }^{39}$. In our experiments, we observe a step-like angular dependence of $V_{\text {ISHE }}$ in both PBTTT and P3HT. Figure 4 (d) shows the observed angle dependence of $V_{\text {ISHE }}$ in PBTTT for various device channel lengths, and a conductivity of $100 \mathrm{~S} / \mathrm{cm}$. It can be well explained by a simple $\cos (\phi)$ dependence (dashed line). The deviations seen in some of the samples are not systematic and are likely due to limited alignment accuracy in the experiments. Figure 4(e) shows a similar step-like angle dependence of $V_{\text {ISHE }}$ in P3HT for various conductivities but a fixed channel length of $L=700$ $\mathrm{nm}$. This result suggests that during diffusion of the spins through the polymer the spin polarisation remains robustly aligned with the direction in which the injected spin current was polarised. 


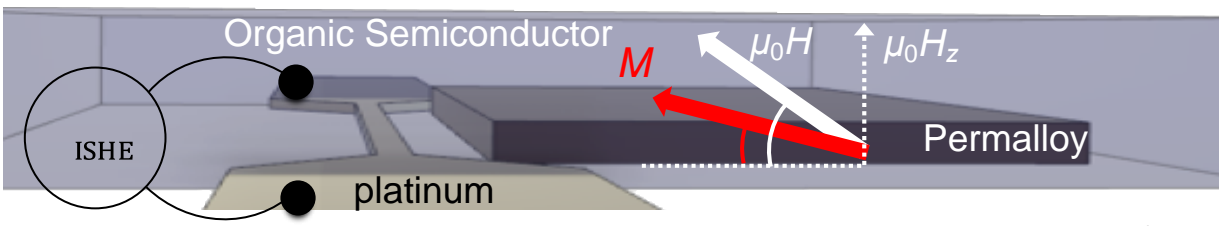

b ${ }_{\text {ISHE }} \propto_{\mathrm{S}} \times$

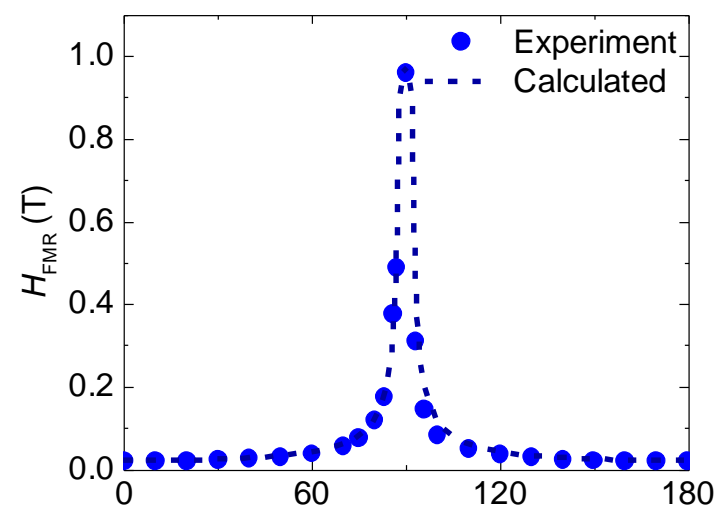

$\left(^{\circ}\right)$

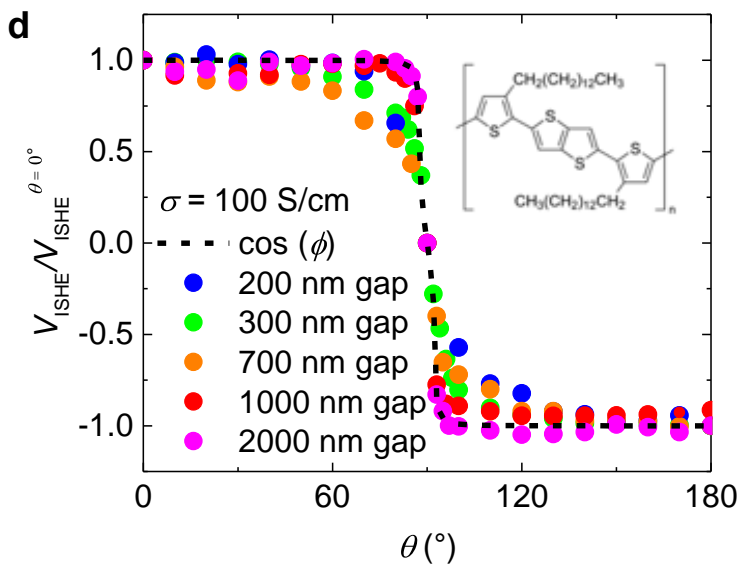

In a microwave field

C

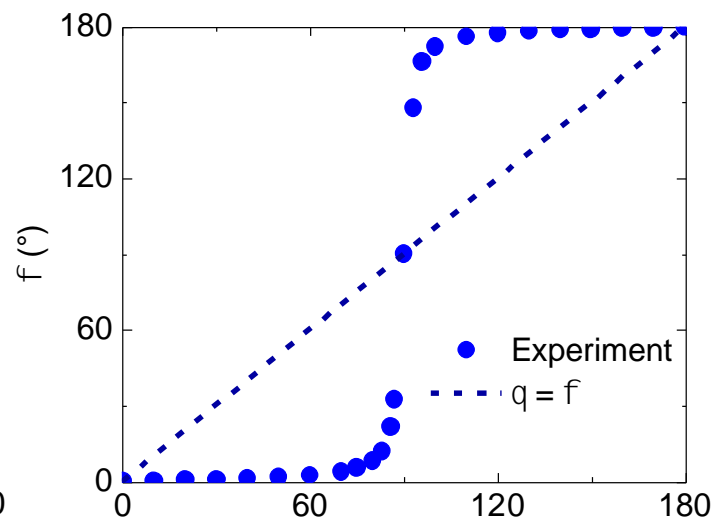

$\left({ }^{\circ}\right)$

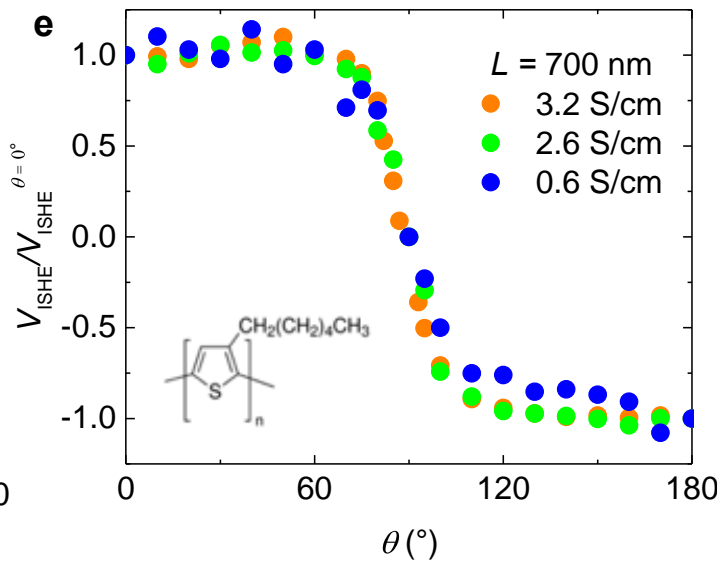

Figure 4 | Angular dependence of the ISHE signal (a) Schematic geometry of Hanle measurements in lateral spin pumping devices (b) FMR resonance field at $4 \mathrm{GHz}$ as a function of the out-of-plane rotation of external magnetic field (c) Difference in angle between the ferromagnet's magnetisation and the direction of applied field during rotation (d) Angle-dependent measurements of VISHE in PBTTT for various channel lengths (e) Angle-dependent

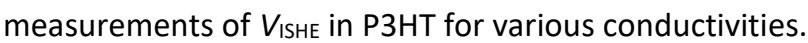

\section{Exchange-mediated spin transport at large carrier densities}

To explain the relatively long spin diffusion length $\left(\lambda_{s}\right)$ and the strong dependence of the ISHE voltage on carrier density, we refer to a recently proposed theoretical framework based on exchange-mediated spin transport in organic semiconductors $4,6,7,8$. This model assumes the 
spin diffusion constant $D$ in the material to have additive contributions from both charge and spin diffusion, with spin diffusion being enhanced at large carrier densities. The carriers are located closer to each other at large carrier densities to enhance the exchange coupling due to the overlap between their wavefunctions, creating an efficient pathway for the transfer of spins between adjacent carriers.

The spin diffusion length is a function of the spin diffusion coefficient $D$ and the spin relaxation time $T_{1}$ through the relation $\lambda_{S}=\sqrt{D T_{1}}$. The spin diffusion coefficient $D$, explicitly written as $D=D_{\text {hop }}+D_{\text {exc }}$, includes charge/spin hopping $\left(D_{\text {hop }}\right)$ and exchange-mediated coupling between spins on adjacent molecules $\left(D_{\text {exc }}\right)$. The latter can propagate spin information without requiring charge motion and is expected to be important only at sufficiently high carrier/spin concentration at which the typical inter-polaron/inter-spin distance $R$ approaches intermolecular distances but can lead to a large enhancement of the diffusion coefficient by several orders of magnitude. The total spin relaxation time $T_{1}$ includes contributions from spin relaxation due to local hyperfine interactions (HFI) and spin-orbit coupling (SOC) fields, i.e., $T_{1}=\left(\omega_{H F I}+\omega_{S O C}\right)^{-1}$. The hopping contribution to the diffusion coefficient depends on the carrier mobility $\mu$ through the Einstein relationship, $D_{\text {hop }}=$ $\mu k_{B} T / e$ (in a more rigorous model, the generalised Einstein relationship ought to be used). We use here a one-dimensional (1D) formulation of the originally 3D model because of the specific microstructure of PBTTT, in which polarons are most likely to come close to each other along the $\pi-\pi$ stacking direction, i.e., we have made explicit modifications to the original 3D expressions for $D_{\text {exc }}$ and $\tau$ used in 8 (SI Section 7). The exchange contribution to the diffusion coefficient takes the form of an isotropic Heisenberg chain in 1D, $D_{\text {exc }}=$ $1.6 J(R) R^{2} / \hbar$ (in SI units), where $J(R)$ is the exchange interaction 57 and may be approximated by a spatially isotropic, hydrogenic exchange interaction of the form $J(R)=$ $0.821 \frac{1}{4 \pi \epsilon \epsilon_{0}}\left(e^{2} / \xi\right)(R / \xi)^{5 / 2} e^{-2 R / \xi}$ (in SI units) as a function of the polaron localisation length $\xi$ and $R^{8,58}$ which gains in strength as the distance between the carriers is reduced. We have computed the polaron localisation length along the $\pi-\pi$ stacking direction to be $\xi=0.4 \mathrm{~nm}$ for PBTTT from first principles. The spin relaxation rate due to the local $\mathrm{HFI}{ }^{8,59}$ is $\omega_{H F I}=$ $2 \Omega_{H F I}^{2} \tau / 3$ where $\Omega_{H F I}$ is the Larmor frequency of the local hyperfine magnetic field and the time $\tau=\left(2 D_{\text {hop }} / a^{2}+2 D_{\text {exc }} / R^{2}\right)^{-1}$ is a measure of the dwell time of a spin on a molecule as it propagates in $1 \mathrm{D}$ along the $\pi-\pi$ stacking direction. $a=0.45 \mathrm{~nm}$ is the average hopping 
distance between polarons along the $\pi-\pi$ stacking direction. The spin relaxation rate due to SOC is $\omega_{S O C}=2 \chi^{2} \tau^{-1}$ where $\chi^{2}=(4 / 3) \gamma^{2}$ is the spin-mixing parameter due to the SOC and $\gamma^{2}$ is calculated from first principles.

Within this model a long spin diffusion length is explained in terms of a large exchangedominated diffusion coefficient. Because the spatial decay length of the exchange interaction is short, a small separation between polarons/spins is necessary for this mechanism to become prominent, i.e., a path of occupied electronic states separated by sufficiently short distances is a prerequisite. This scenario is achieved at large doping concentrations (large $n$ and small $R$, where $R=1 / \sqrt[3]{n}$ ). A second requirement for a long spin diffusion length is that the $T_{1}$ is sufficiently long. Density-functional theory calculations of the hyperfine field strength in the polymer units revealed weak fields of $<10$ gauss, indicating a dominance of the SOC spin relaxation mechanism over HFI (SI Section 7). The electronic states in a semiconductor are a mix of spin-up and spin-down states on account of which an electron hopping through a polymer network loses spin polarisation through the Elliott-Yafet (EY) mechanism ${ }^{60,61}$. A long spin relaxation time $T_{1}$ therefore requires a low SOC, in particular a small value of $\gamma^{2}$.

Although the polymers chosen in this study have relatively large mobilities between 0.1 (for $\mathrm{P} 3 \mathrm{HT}$ ) and $1 \mathrm{~cm}^{2} / \mathrm{Vs}$ (for PBTTT), at large carrier concentrations the exchange diffusion coefficient $D_{\text {exc }}$ dominates the overall diffusion coefficient $D$ by two orders of magnitude as shown in Fig. 5(a). In the model both $n$ and $\gamma^{2}$ impact the spin relaxation time $T_{1}$, while $n$ impacts $D$ through the exchange integral $J(R)$. Fig. $5(\mathrm{~b})$ shows the calculated spin diffusion length $\lambda_{S}$ plotted as a function of carrier density $n$ under the assumption of constant carrier mobility. In the regime where the diffusion coefficient is dominated by the exchange mechanism, the theoretically predicted spin diffusion length achieves values of over $1 \mu \mathrm{m}$. This prediction is matched by the experimentally determined values of $\lambda_{S}$ shown in Fig. 5 (b). The experimental value of the carrier density for the highest conductivity in PBTTT and P3HT were measured using ESR and taken together with the measured conductivity of $100 \mathrm{~S} / \mathrm{cm}$ for PBTTT, the mobility was extracted to be $\sim 4 \mathrm{~cm}^{2} / \mathrm{Vs}$ using $\sigma=n e \mu$. This carrier mobility, although high, is not unwarranted seeing as the distance between the injector and the detector is a few hundred nanometres, thus potentially reflecting intra-grain transport. The carrier densities of the other measured data points shown in Fig. 5(b) were estimated from 
the measured conductivities, which depend on doping concentration, using the mobility determined by ESR at the highest carrier density. The experimental data of Fig. 5(b) clearly tracks the decline in the spin diffusion length as the exchange interaction is weakened due to increasing distances between polarons/spins on de-doping the film, providing strong evidence for exchange-mediated spin diffusion being the mechanism responsible for the long spin diffusion length observed here. We also find that the predicted exchange diffusion coefficient on the order of $1 \mathrm{~cm}^{2} / \mathrm{s}$ is consistent with the measured $\lambda_{S} \approx 1.2 \mu \mathrm{m}$ and the value of $T_{1} \approx 22$ ns determined independently by ESR (SI Section 6 ) according to $\lambda_{S}=\sqrt{D T_{1}}$.

To explain the difference in spin diffusion length between P3HT and PBTTT, which have similar carrier densities and $\pi-\pi$ stacking distances, we consider differences in the SOC strength 4,62 . The parameter $\gamma^{2}$ in a conjugated molecule increases when the adjacent units are not coplanar but exhibit finite torsion angles between each other ${ }^{62}$. In PBTTT, we expect maximal spin diffusion lengths to be determined by virtually flat polymer chains, corresponding to a low $\gamma^{2}$. In P3HT, literature suggests a variation of thiophene - thiophene dihedral angles between 20 - 30 degrees, and 40 - 60 degrees in comparable polymer samples ${ }^{63,55,64}$. We consequentially calculate $\gamma^{2}$ for representative geometries in these two limits. Fig. 5(c) shows the joint dependence of the spin diffusion length $\lambda_{\mathrm{S}}$ on $n$ and on the up-down spin admixture parameter $\gamma^{2}$ that reflects the strength of SOC. $\lambda_{\mathrm{S}}$ is nearly inversely proportional to $\gamma$, and increases with increasing $n$.

The accuracy of our theoretical spin diffusion length predictions is surprisingly good given the simplicity of the model. We emphasize that all model parameters have been taken either directly from experiments or calculated from electronic structure theory and adapted to (effectively) 1D transport corresponding to our understanding of the actual spin dynamics in the polymer samples. However, it is possible that the good agreement is at least partly due to cancellation of errors/inaccuracies of the model. On the one hand, test calculations indicate that charge motion at intermediate polaron concentrations may significantly contribute to spin diffusion. This effect is neglected in a static spin diffusion model, therefore underestimating the predicted spin diffusion lengths. On the other hand, the exchange coupling expression derived in 8 - even with a polaron delocalisation length estimated from first-principles as used here - is significantly stronger than frequently used expressions ${ }^{65}$ at 
precisely the same intermediate spin concentrations, which appears to cancel the error due to lack of dynamic effects. There is clearly a need for a more microscopic model of spin diffusion in such systems.

\section{Observed absence of spin precession during spin transport}

Finally, we would like to discuss possible reasons why the spin polarisation remains robustly aligned with the magnetisation direction and does not appear to precess around the external magnetic field, as the spins diffuse through the organic layer over micrometer distances. One possible interpretation of this result is that the spins transit through the polymer on a timescale $\tau_{T}$ that is faster than the timescale $\tau_{L}$ for precession in the external magnetic field. It is tempting to interpret the absence of the Hanle effect as a characteristic feature of rapid exchange-enhanced spin diffusion as it was in fact claimed theoretically ${ }^{8}$ to explain the absence of a Hanle signature in vertical organic spin valves ${ }^{23,66}$. In our system, the exchangeenhanced spin transit time $\tau_{T} \approx L^{2} / D_{\text {exc }}$ is indeed very fast, on the order of 10 ns. However, it remains comparable to $\tau_{L} \approx 2 \pi / \gamma_{e} B_{\text {ext }}^{\perp}$, where $\gamma_{e}$ is the electron gyromagnetic ratio and $B_{\text {ext }}^{\perp}$ is the component of the external field perpendicular to the magnetisation. Using a simplified model of the experiment discussed in SI Section 6, we argue that spin precession should have manifested itself in a more gradual angular dependence than what is observed experimentally, unless $D_{\text {exc }}$ was in fact even higher than predicted by the exchange-based spin diffusion model. This seems unlikely because the relationship $\lambda_{S}=\sqrt{D T_{1}}$ puts a bound on $D_{\text {exc }}$ values to be compatible with the measured values of $\lambda_{S}$ and $T_{1}$. A second, more likely, interpretation is that it is in fact an inherent feature of the lateral spin pumping architecture. It is worth noting that in carefully performed experiments on Si and Graphene, where it was possible to perform both lateral spin pumping and non-local electrical spin injection and detection measurements and establish that both experiments yield consistent and reliable values for the spin diffusion length, a Hanle signature was observed only in the nonlocal spin valve but not in the lateral spin pumping architecture ${ }^{42,44}$. In fact, none of the lateral spin pumping experiments reported to date have shown a gradual angular dependence (SI Section 4). In the simplified model of SI Section 6 we have neglected the fact that in the lateral spin pumping experiment the injected spin polarisation has not just a DC component but also an 
AC component, which is driven by the $A C$ microwave field and is precessing around the magnetisation direction. It is possible that in the rotating frame of reference of the precessing spins a DC applied magnetic field is very ineffective in re-orientating the axis of the spin precession and that the axis of precession is kept aligned by the AC microwave field. In fact, theoretical considerations have argued that the driving AC microwave can even cause a spin rectification effect that enhances the DC component of the spin polarisation along the magnetisation direction ${ }^{67}$. To observe a signature of Hanle precession in the lateral spin pumping architecture may require more challenging $\mathrm{AC}$ measurements, which go beyond the scope of the present work, whose focus is on explaining the exceptionally long spin diffusion lengths observed in doped conjugated polymers. 
a

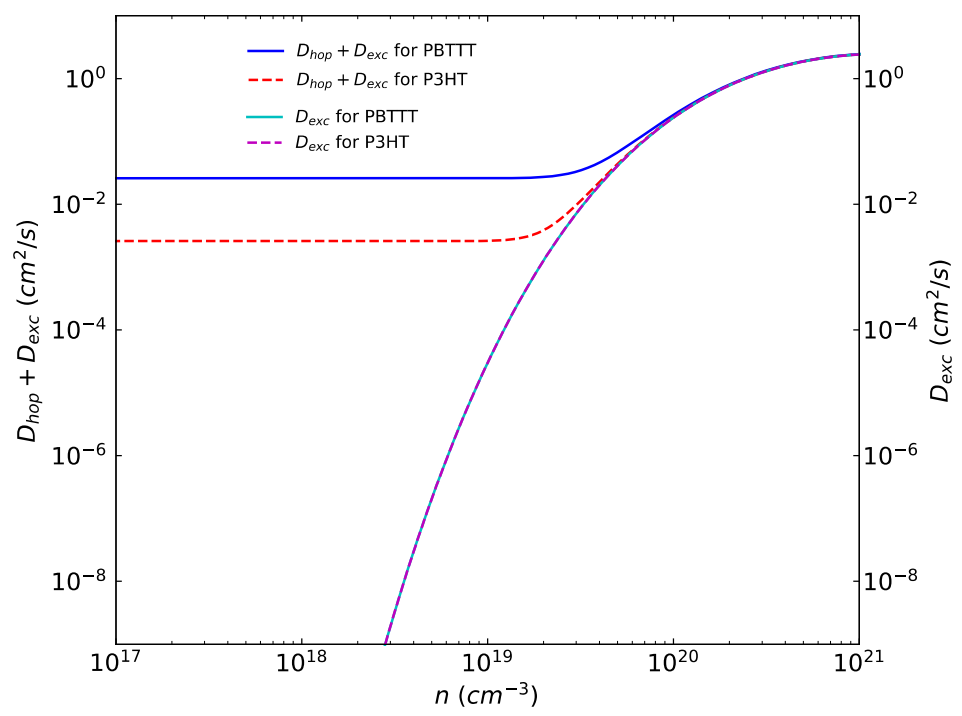

b

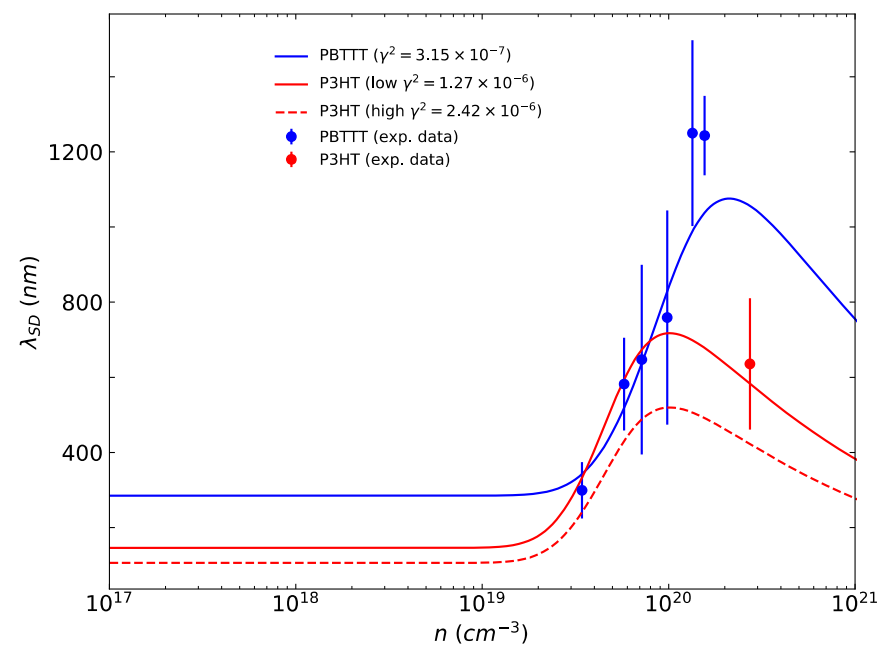

C

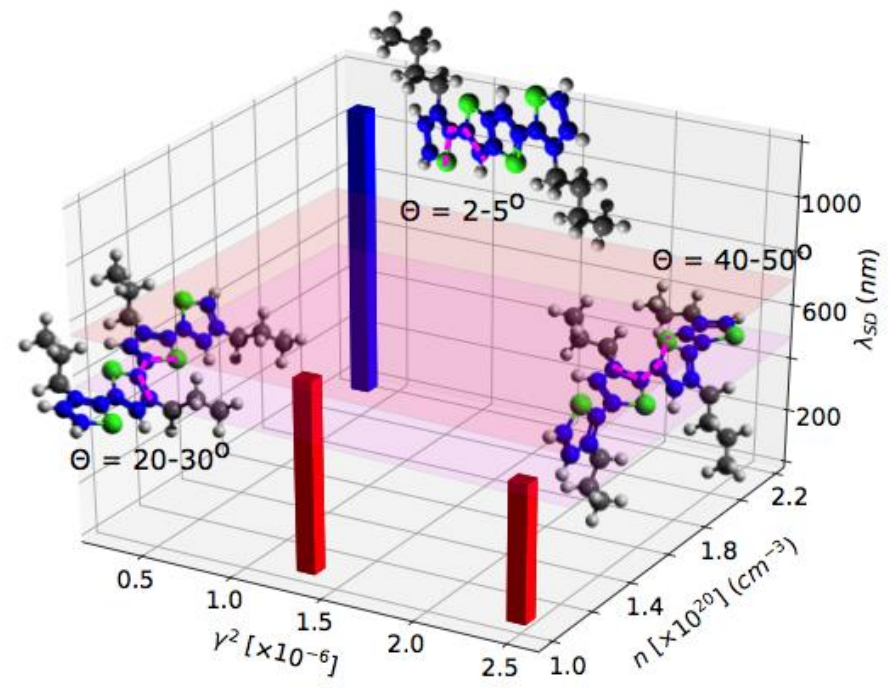

Figure 5 | Theoretical modelling of spin transport in an exchange mediated regime. (a) Total diffusion coefficient that includes contributions from hopping and exchange as a function of carrier density for PBTTT and 
P3HT. (b) Spin diffusion length as a function of carrier density for PBTTT and P3HT. The maximum spin diffusion length for PBTTT is $1.102 \mu \mathrm{m}$ at a theoretically predicted $n=1.91 \times 10^{20} \mathrm{~cm}^{-3}$. For P3HT with low value of spinmixing parameter $\gamma^{2}=1.27 \times 10^{-6}$, it is $0.72 \mu \mathrm{m}$ and with a high value of $\gamma^{2}=2.42 \times 10^{-6}$, it is $0.52 \mu \mathrm{m}$ at a theoretically predicted $n=1.01 \times 10^{20} \mathrm{~cm}^{-3}$. (c) The spin diffusion length for PBTTT and P3HT shown as a function of both $\gamma^{2}$ and $n$. A variation in $\gamma^{2}$ is caused by different dihedral angles in the molecule.

\section{Conclusions}

We have explored spin diffusion in doped organic polymers using a combination of experiment and theory. Long spin diffusion lengths of more than $1 \mu \mathrm{m}$ and fast spin transit times on the order of $10 \mathrm{~ns}$ are achievable in the presence of a sufficiently high spin density (on the order of $10^{20} \mathrm{~cm}^{-3}$ ) such that the exchange mechanism decouples charge and spin transport and opens up a pathway for fast spin motion down a track with a sufficiently small average separation between sites. In this unique spin transport regime, the spin diffusion length is limited by SOC strength at hopping sites via the EY spin relaxation mechanism. The reported long spin diffusion lengths and large diffusion coefficients on the order of $1 \mathrm{~cm}^{2} / \mathrm{s}$ in doped organic polymers are comparable to other well understood spintronic materials such as GaAs, Graphene and Silicon (Si has a diffusion coefficient of $1.8 \mathrm{~cm}^{2} / \mathrm{s}^{42}$ ) with organic materials offering an added advantage of chemical tunability and comparatively long spin lifetimes. The efficient exchange-mediated spin transport mechanism opens up new prospects for doped conjugated polymers in spintronic applications, including low-power, spin-current based information processing and sensing. 


\section{Bibliography}

1. Cinchetti, M., Dediu, V. \& Hueso, L., Activating the molecular spinterface. Nature Materials 16, 507-515 (2017).

2. Warner, M. et al., Potential for spin-based information processing in a thin-film molecular semiconductor. Nature 503, 504 (2013).

3. Dediu, V., Hueso, L., Bergenti, I. \& Taliani, C., Spin routes in organic semiconductors. Nature Materials 8, 707-716 (2009).

4. Yu, Z. G., Spin-Orbit Coupling, Spin Relaxation, and Spin Diffusion in Organic Solids. Physical Review Letters 106, 106602 (2011).

5. Sinova, J., Valenzuela, S. O., Wunderlich, J., Back, C. H. \& Jungwirth, T., Spin Hall effects. Reviews of Modern Physics 87, 1213 (2015).

6. Yu, Z. G., Spin transport and the Hanle effect in organic spintronics. De Gruyter Open: Nanoelectronics and Spintronics 1, 1-18 (2015).

7. Yu, Z. G., Impurity-band transport in organic spin valves. Nature Communications 5, 4842 (2014).

8. Yu, Z. G., Suppression of the Hanle Effect in Organic Spintronic Devices. Physical Review Letters 111, 016601 (2013).

9. Lou, X. et al., Electrical detection of spin transport in lateral ferromagnet-semiconductor devices. Nature Physics 3, 197-202 (2007).

10. Sirringhaus, H., 25th Anniversary Article: Organic Field-Effect Transistors: The Path Beyond Amorphous Silicon. Advanced Materials 26, 1319-1335 (2014).

11. Xiong, Z. H., Wu, D., Vardeny, Z. V. \& Shi, J., Giant magnetoresistance in organic spin-valves. Nature 427, 821-824 (2004).

12. Nguyen, T. D. et al., Isotope effect in spin response of $\pi$-conjugated polymer films and devices. Nature Materials 9, 345-352 (2010).

13. Zhang, X. et al., Observation of a large spin-dependent transport length in organic spin valves at room temperature. Nature Communications 4, 1392 (2013).

14. Mooser, S., Cooper, J. F. K., Banger, K. K., Wunderlich, J. \& Sirringhaus, H., Spin injection and transport in a solution-processed organic semiconductor at room temperature. Physical Review B 85, 235202 (2012).

15. Majumdar, S. \& Majumdar, H. S., Decay in spin diffusion length with temperature in organic semiconductors-An insight of possible mechanisms. Organic Electronics 173, $26-30$ (2013). 
16. Li, F., Li, T., Chen, F. \& Zhang, F., Excellent spin transport in spin valves based on the conjugated polymer with high carrier mobility. Scientific Reports 5, 9355 (2015).

17. Sasaki, T. et al., Temperature dependence of spin diffusion length in silicon by Hanle-type spin precession. Applied Physics Letters 96, 122101 (2010).

18. Jain et al., A., Crossover from Spin Accumulation into Interface States to Spin Injection in the Germanium Conduction Band. Physical Review Letters 109, 106603 (2012).

19. Drögeler, M. et al., Spin Lifetimes Exceeding 12 ns in Graphene Nonlocal Spin Valve Devices. Nano Letters 16, 3533-3539 (2016).

20. Yan, W. et al., Long Spin Diffusion Length in Few-Layer Graphene Flakes. Physical Review Letters 117, 147201 (2016).

21. Szulczewski, G., Sanvito, S. \& Coey, M., A spin of their own. Nature Materials 8, 693 (2009).

22. Grünewald, M. et al., Tunneling anisotropic magnetoresistance in organic spin valves. Physical Review B 84, 125208 (2011).

23. Grünewald, M. et al., Vertical organic spin valves in perpendicular magnetic fields. Physical Review B 88, 085319 (2013).

24. Jedema, F. J., Heersche, H. B., Filip, A. T., Baselmans, J. J. A. \& van Wees, B. J., Electrical detection of spin precession in a metallic mesoscopic spin valve. Nature 416, 713-716 (2002).

25. Olejnik, K. et al., Detection of Electrically Modulated Inverse Spin Hall Effect in an Fe/GaAs Microdevice. Physical Review Letters 109, 076601 (2012).

26. Avsar, A. et al., Toward Wafer Scale Fabrication of Graphene Based Spin Valve Devices. Nano Letters 11 (6), 2363-2368 (2011).

27. Zhou, Y. et al., Electrical spin injection and transport in germanium. Physical Review B 84, 125323 (2011).

28. Salis, G., Fuhrer, A., Schlittler, R., Gross, L. \& Alvarado, S., Temperature dependence of the nonlocal voltage in an Fe/GaAs electrical spin-injection device. Physical Review B 81, 205323 (2010).

29. Fujiwara, K. et al., $5 \mathrm{~d}$ iridium oxide as a material for spin-current detection. Nature Communications 4, 2893 (2013).

30. Kamiya, T., Kawasugi, Y., Ara, M. \& Tada, H., Nonlocal magnetoresistance measurements of the organic zero-gap conductor $\alpha-(B E D T-T T F) \_2$ I_3. Physical Review B 95, 085307.

31. Tombros, N., Jozsa, C., Popinciuc, M., Jonkman, H. T. \& van Wees, B. J., Electronic spin transport and spin precession in single graphene layers at room temperature. Nature 448, 571-574 (2007). 
32. Schmidt, G., Ferrand, D., Molenkamp, L. W., Filip, A. T. \& van Wees, B. J., Fundamental obstacle for electrical spin injection from a ferromagnetic metal into a diffusive semiconductor. Physical Review B 62, R4790 (2000).

33. Martin, S. et al., Flicker noise properties of organic thin-film transistors. Journal of Applied Physics 87, 3381 (2000).

34. Jang, H.-J. \& Richter, C. A., Organic Spin-Valves and Beyond: Spin Injection and Transport in Organic Semiconductors and the Effect of Interfacial Engineering. Advanced Materials 29, 1602739 (2017).

35. de Oliveira, T. V. A. G., Gobbi, M., Porro, J. M., Hueso, L. E. \& Bittner, A. E., Charge and spin transport in PEDOT:PSS nanoscale lateral devices. Nanotechnology 24 (47), 475201 (2013).

36. Tserkovnyak, Y., Brataas, A. \& Bauer, G. E. W., Enhanced Gilbert Damping in Thin Ferromagnetic Films. Physical Review Letters 88, 117601 (2002).

37. Silsbee, R. H., Janossy, A. \& Monod, P., Coupling between ferromagnetic and conduction-spinresonance modes at a ferromagnetic-normal-metal interface. Physical Review B 19, 4382 (1979).

38. Watanabe, S. et al., Polaron spin current transport in organic semiconductors. Nature Physics 10, 308 (2014).

39. Jiang, S. W. et al., Exchange-Dominated Pure Spin Current Transport in Alq3 Molecules. Physical Review Letters 115, 086601 (2015).

40. Mendes, J. B. S. et al., Efficient spin transport through polyaniline. Physical Review B 95, 014413 (2017).

41. Maekawa, S., Valenzuela, S. O., Saitoh, E. \& Kimura, T., Spin Current (Oxford University Press, Oxford, 2012).

42. Shikoh, E. et al., Spin-Pump-Induced Spin Transport in p-Type Si at Room Temperature. Physical Review Letters 110, 127201 (2013).

43. Yamamoto, A., Ando, Y., Shinjo, T., Uemura, T. \& Shiraishi, M., Spin transport and spin conversion in compound semiconductor with non-negligible spin-orbit interaction. Physical Review B 91, 024417 (2015).

44. Tang, Z. et al., Dynamically generated pure spin current in single-layer graphene. Physical Review B 87, 140401(R).

45. Dushenko, S. et al., Experimental Demonstration of Room-Temperature Spin Transport in nType Germanium Epilayers. Physical Review Letters 114, 196602 (2015).

46. Yamamoto, T., Seki, T., Ono, S. \& Takanashi, K., Characterization of spin pumping effect in Permalloy/Cu/Pt microfabricated lateral devices 115, 17C505 (2014).

47. Ohshima, R. et al., Strong evidence for d-electron spin transport at room temperature at a LaAlO3/SrTiO3 interface. Nature Materials 16, 609-614 (2017). 
48. Tserkovnyak, Y., Brataas, A. \& Bauer, G. E. W., Spin pumping and magnetization dynamics in metallic multilayers. Physical Review B 66, 224403 (2002).

49. Brataas, A., Tserkovnyak, Y., Bauer, G. \& Halperin, B., Spin battery operated by ferromagnetic resonance. Physical Review B 66, 060404(R) (2002).

50. Ando, K. \& Saitoh, E., Observation of the inverse spin Hall effect in silicon. Nature Communications 3, 629 (2012).

51. Saitoh, E., Ueda, M., Miyajima, H. \& Tatara, G., Conversion of spin current into charge current at room temperature: Inverse spin-Hall effect. Applied Physics Letters 88, 182509 (2006).

52. McCulloch, I. et al., Liquid-crystalline semiconducting polymers with high charge-carrier mobility. Nature Materials 5, 328-333 (2006).

53. Schuettfort, T. et al., Microstructure of Polycrystalline PBTTT Films: Domain Mapping and Structure Formation. ACS Nano 6 (2), 1849-1864 (2012).

54. Kang, K. et al., 2D coherent charge transport in highly ordered conducting polymers doped by solid state diffusion. Nature Materials 15, 896 (2016).

55. Alberga, D. et al., Morphological and charge transport properties of amorphous and crystalline P3HT and PBTTT: insights from theory. Physical Chemistry Chemical Physics 17, 18742 (2015).

56. Tani, Y., Kondo, T., Teki, Y. \& Shikoh, E., Spin current relaxation time in thermally evaporated pentacene films. Applied Physics Letters 110, 032403 (2017).

57. Morita, T., Spin Diffusion in the Heisenberg Magnets at Infinite Temperature 6, 3385 (1972).

58. Herring, C. \& Flicker, M., Asymptotic Exchange Coupling of Two Hydrogen Atoms. Phys. Rev. 134, A362 (1964).

59. Yu, Z. G., Ding, F. \& Wang, H., Hyperfine interaction and its effects on spin dynamics in organic solids. Physical Review B 87 (20), 205446 (2013).

60. Elliott, R. J., Theory of the Effect of Spin-Orbit Coupling on Magnetic Resonance in Some Semiconductors. Physical Review 96 (2), 266-279 (1954).

61. Yafet, Y., in Solid State Physics (F. Seitz and D. Turnbull, Eds. Academic, New York, 1963), pp. 198.

62. Yu, Z. G., Spin-orbit coupling and its effects in organic solids. Physical Review B 85 (11), 115201 (2012).

63. Boeckmann, M. et al., Structure of P3HT crystals, thin films, and solutions by UV/Vis spectral analysis. Physical Chemistry Chemical Physics 17, 28616 (2015).

64. Luzny, W., X-ray diffraction and computer modelling study of the structure and conformation of poly(3-decylthiophene). Acta Crystallogr. Sect. B Struct. Sci. 51 (2), 255-260 (1995). 
65. O'Dea, A. R., Curtis, A. F., Green, N. J. B., Timmel, C. R. \& Hore, P. J., Influence of Dipolar Interactions on Radical Pair Recombination Reactions Subject to Weak Magnetic Fields. Journal of Physical Chemistry A 109 (5), 869-873 (2005).

66. Riminucci, A. et al., Hanle effect missing in a prototypical organic spintronic device. Applied Physics Letters 102, 092407 (2013).

67. Sakimura, H., Matsumoto, T. \& Ando, K., Spin rectification induced by dynamical Hanle effect. Applied Physics Letters 103, 132402 (2013). 


\section{Authors Contributions}

SJW, DV, HS and JW developed the idea of probing spin transport in organic semiconductors using lateral spin pumping architectures. SJW and DV nanofabricated the devices and performed lateral spin pumping measurements. MRM, UC, ERM, SAE, SM, SS and JS developed the supporting theory. RDP and AW setup the experimental facilities required to do the experiments. RDP, JW, DV conceived the ideas for control experiments to remove spurious artefacts. SJW, GS, KHK, IEJ developed the doping techniques used. RC and SS performed ESR measurements. DPGHW and SS performed the supporting Hanle simulations. $\mathrm{CJ}, \mathrm{ML}, \mathrm{AM}$ and IM synthesised and characterised the polymers used. MC, JNMS, TJW, OZ and PS provided technical help with measurements. DV nanofabricated and measured organic nonlocal spin valves. ROA and Al assisted with thin film deposition of ferromagnets and tunnel barriers for organic nonlocal spin valves. DV, SJW, ERM and HS wrote the manuscript with inputs from the other authors. All authors within the ERC Synergy SC2 grant made significant contributions to discussions throughout the progression of the project.

\section{Acknowledgements}

The authors wish to thank Dr Laurent Vila and Dr Stephane Auffret from SPINTEC in France for support with the fabrication of $\mathrm{Co} / \mathrm{Al}_{2} \mathrm{O}_{3}$ films used in the organic nonlocal spin valves. We also thank Prof Crispin H W Barnes and Dr Spencer John Brennan from the Thin Film Magnetism group of the Cavendish Laboratory for support with metal deposition, and Dr James A. Haigh of Hitachi Cambridge Laboratories for discussions on the measurements. Prof. Klaus Müllen of MPI Mainz supplied the polymer CDT-BTZ, for which the authors are grateful. The inputs of Ms Chen Chen and Mr John Armitage on the doping of organic semiconductors is also gratefully acknowledged. DV is thankful to Mr. Xiao-Jian She for discussions on orthogonal resists. G.S. acknowledges postdoctoral fellowship support from the WienerAnspach Foundation and the Leverhulme Trust (Early Career Fellowship supported by the Isaac Newton Trust). I.E.J. acknowledges funding from the Royal Society through a Newton International Fellowship. Finally, the authors are very grateful for the excellent technical support offered by Dr Radoslav Chakalov and Mr Roger Beadle during the course of the ERC Synergy grant. Funding from the ERC Synergy Grant SC2 (Grant No. 610115) is gratefully acknowledged.

\section{Methods}




\section{Device Fabrication}

The lateral spin pumping (LSP) devices were prepared using a multiple step e-beam lithography and metal deposition process on $5 \mathrm{~mm} \times 3 \mathrm{~mm} \mathrm{Si} / \mathrm{SiO}_{2}(300 \mathrm{~nm})$ substrates. The Pt wire $(10 \mathrm{~nm})$ was deposited by magnetron sputtering at a base pressure of $5 \times 10^{-7} \mathrm{mbar}$ at a rate of $5 \AA / s$. The Py island $(25 \mathrm{~nm})$ was deposited by e-beam evaporation at a base pressure of $2 \times 10^{-7} \mathrm{mbar}$ with a rate of $2 \AA / \mathrm{s}$. The organic semiconductors were then spin coated from solution onto the devices. PBTTT $(80 \mathrm{~nm}$ ) was spin coated from a $10 \mathrm{mg} / \mathrm{ml} \mathrm{DCB}$ solution (maintained at $80^{\circ} \mathrm{C}$ ) at $5000 \mathrm{rpm}$ and annealed at $180^{\circ} \mathrm{C}$ for 20 minutes to achieve a terraced phase. P3HT $(80 \mathrm{~nm})$ was spin coated from unheated $10 \mathrm{mg} / \mathrm{ml} \mathrm{DCB}$ solution at $5000 \mathrm{rpm}$. The $\mathrm{P} 3 \mathrm{HT}$ film was annealed at $110^{\circ} \mathrm{C}$ for 20 minutes to improve the microstructure and charge transport properties. The organic semiconductors were doped by F4TCNQ via a sequential doping method where the films were immersed in $1.5 \mathrm{mg} / \mathrm{ml} \mathrm{F4TCNQ}$ acetonitrile solution. Following doping, samples were annealed at $80^{\circ} \mathrm{C}$ to achieve a uniform distribution of dopant molecules. The organic semiconductor films were then patterned mechanically, and contact was made to the Pt wire using Ag paste. For PBTTT based LSP devices doped to $100 \mathrm{~S} / \mathrm{cm}$, the device resistance was $3.1 \mathrm{kOhm}$. When de-doped to $17 \mathrm{~S} / \mathrm{cm}$, the device resistance was $12.5 \mathrm{kOhm}$. For P3HT based LSP devices at $3.2 \mathrm{~S} / \mathrm{cm}$, the device resistance was $17.5 \mathrm{kOhm}$, at $0.3 \mathrm{~S} / \mathrm{cm}$ it changed to $25.3 \mathrm{kOhm}$.

\section{Spin pumping measurements}

Ferromagnetic resonance (FMR) of Py was excited in either an electron spin resonance (ESR) system or a coplanar waveguide setup. Both methods for exciting FMR gave similar results. For measurements in the ESR setup (Bruker E500 X-band spectrometer), the sample was placed in the centre of a Bruker ER 4122SHQE cavity at a microwave frequency of $9.38 \mathrm{GHz}$. Two ends of the Pt wire were connected to a KEITHLEY 2182A Nanovoltmeter with Ag paste and $\mathrm{Cu}$ wires to detect the voltage due to the Inverse Spin Hall Effect. For measurements using a coplanar waveguide, the sample was flipped face down onto the coplanar waveguide (to ensure proximity with the strip line) and a layer of polyamide isolated the sample from the waveguide. Both ends of the Pt wire were connected to a KEITHLEY 2182A Nanovoltmeter using Ag paste. Low temperature spin pumping measurements were performed by positioning the coplanar waveguide in a Helium exchange gas cryostat with microwave access. 


\section{Data Availability}

The authors declare that all relevant data are included in the paper and in the accompanying Supplementary Information. Additional data are available from the corresponding authors upon reasonable request. 